\title{
Distribuição otimizada do reforço com fibras de carbono em lajes de concreto armado
}

\author{
Optimal distribution of carbon-fiber reinforcement in \\ concrete slabs
}

\begin{abstract}
Jesiel Cunha
Luciano Pereira Chaves

Leila Maria Soares dos Santos

Zigue Marley Furtado Lopes

Resumo

$\mathbf{N}$

este trabalho é determinada a região de aplicação do reforço com fibras de carbono em lajes de concreto utilizando-se um procedimento de otimização topológica. Embora o procedimento seja aplicado em lajes, a técnica pode ser usada em qualquer estrutura de concreto armado a ser reforçada. As simulações numéricas foram feitas através do Método dos Elementos Finitos, associadas ao procedimento automático de otimização topológica, para indicação da região ótima de posicionamento do reforço. A influência de alguns aspectos do comportamento estrutural da laje no resultado da otimização é apresentada: fissuração do concreto; condições de apoio e de carregamento; e taxa de reforço. Foram verificados os ganhos em termos de rigidez e de resistência das peças reforçadas. A comparação com técnicas convencionais de reforço mostrou que a otimização topológica pode ser uma ferramenta bastante útil para a definição da região do reforço, podendo levar a uma economia de material.

Jesiel Cunha Universidade Federal de Uberlândia Uberlândia - MG - Brasil

Luciano Pereira Chaves Universidade Federal de Uberlândia Uberlândia - MG - Brasil

Leila Maria Soares dos Santos Universidade de Cabo Verde Santiago - Cabo Verde

Zigue Marley Furtado Lopes Universidade de Cabo Verde Santiago - Cabo Verde

Palavras-chaves: Reforço estrutural. Otimização topológica. Fibras de carbono. Materiais compostos. Lajes de concreto.

\section{Abstract}

This study proposes a topology optimization procedure as a tool to find the region of application of carbon-fiber reinforcement in concrete slabs. Although the procedure is applied to slabs, the technique can be used for the reinforcement of any concrete structure. Numerical simulations were performed using the Finite Element Method in combination with an automated topology optimization procedure in order to indicate the optimal region for the placement of the reinforcement. The influence of some aspects of the structural behavior of the slab on the optimization results is presented: concrete cracking, load and support conditions and reinforcement rates. Gains were found in the stiffness and strength of the reinforced structures. A comparison with conventional reinforcement techniques demonstrates that topology optimization can be a useful tool for determining the region of reinforcement, allowing material cost savings.
\end{abstract}

Recebido em 04/04/14 Aceito em 01/03/15
Keywords: Structural strengthening. Topology optimization. Carbon fibers. Composite materials. Concrete slabs. 


\section{Introdução}

Existem várias razões para se intervir em uma estrutura de concreto armado:

(a) mudança no uso da edificação, com aumentos de carga;

(b) ocorrência de danos em partes da estrutura; reabilitação após sinistros;

(c) ocorrência de erros de projeto e de execução da estrutura; modificação do sistema estrutural, com a criação, por exemplo, de aberturas em lajes; $\mathrm{e}$

(d) mudanças nas normas técnicas, com novas exigências (HOLLAWAY; HEAD, 2001).

$\mathrm{Na}$ maioria dessas ocorrências, o reforço estrutural é uma solução viável. As técnicas de reforço estrutural desenvolvidas nas últimas décadas consistem basicamente na adição de elementos estruturais à face externa das peças, segundo as direções preferenciais dos esforços. Tradicionalmente, os elementos estruturais onde o reforço pode ser aplicado são vigas, pilares, alvenarias, muros de arrimo, vigas-parede, reservatórios, silos, chaminés, túneis, tubulações, entre outros.

Em contraponto ao reforço tradicional, que utiliza principalmente chapas de aço, as vantagens da técnica de reforço com fibras de carbono são a alta resistência e a alta rigidez do material, possibilitando a utilização de reforço com baixa espessura e baixo peso, o que gera pequeno acréscimo de carga permanente. Além disso, destaca-se a flexibilidade das mantas de fibra de carbono, que se adaptam a qualquer forma, sendo de execução simples, precisa e rápida, com mão de obra reduzida. O material exige pouca manutenção e, além disso, é altamente resistente à corrosão e, portanto, possui grande durabilidade.

Uma dificuldade para o projeto da estrutura reforçada consiste em definir a melhor forma de distribuir as mantas ou faixas de fibras de carbono na superfície da peça. Normalmente se recorre à experiência, à facilidade construtiva e ao princípio de funcionamento estrutural do elemento reforçado. No entanto, para geometrias complexas das peças e para condições de contorno e de carregamento diferenciadas, a escolha da melhor distribuição do reforço não é evidente. A determinação de uma configuração de reforço eficiente em uma estrutura é habitualmente associada a um processo de "tentativa e erro", cujo resultado não garante com segurança que tenha sido encontrado o projeto ótimo. A otimização matemática, nestes casos, pode ser uma ferramenta interessante para encontrar soluções mais eficientes. Em particular, a otimização estrutural busca a criação de estruturas com melhor desempenho e menor consumo de material, o que reduz os custos.

Em um problema de otimização estrutural deve-se definir uma ou mais funções objetivo, sujeitas ou não a restrições, que sejam capazes de gerar um projeto que atenda às condições de resistência e rigidez, com o menor custo. Neste sentido, surgiu a otimização topológica, em face da necessidade de se aprimorar a otimização de forma (contorno da estrutura), permitindo melhor distribuição de material no interior do domínio de projeto, ou seja, no interior da estrutura que sofrerá mudança de sua geometria. A distribuição topológica ótima do material está associada a um objetivo, que pode ser, por exemplo, a minimização do volume final da estrutura, visando à economia de material, ou à maximização da rigidez, ou ainda à maximização da resistência da peça. Diferentemente da otimização de forma, na otimização topológica furos podem ser criados no domínio durante o processo de otimização. Assim, a otimização topológica permite obter uma nova configuração da estrutura.

$\mathrm{Na}$ literatura existem poucos trabalhos que utilizam a otimização topológica para gerar configurações geométricas ótimas de estruturas de concreto (BRUGGI, 2009; GUAN et al., 2003). Os trabalhos sobre o reforço de lajes com fibras de carbono são de ordem experimental e numérica, em que se utiliza o Método dos Elementos Finitos e soluções analíticas desenvolvidas em séries de Fourier (MOSALLAM; MOSALAM, 2003; AGBOSSOU et al., 2008; MICHEL et al., 2009; EBEAD; MARZOUK; LYE, 2002). Não foi encontrado trabalho que apresente um procedimento de otimização topológica para auxílio à distribuição eficaz do reforço nas faces das peças.

O objetivo deste trabalho é estudar a melhor distribuição do reforço com fibras de carbono em lajes maciças de concreto armado, a partir da necessidade de aumento da capacidade de carga, usando a otimização topológica como ferramenta para definir as regiões onde será posicionado o reforço. Embora o procedimento seja aplicado neste trabalho em lajes, a técnica pode ser usada em qualquer estrutura de concreto armado a ser reforçada. As simulações numéricas foram feitas pelo Método dos Elementos Finitos simultaneamente ao procedimento automático de otimização topológica. Foi considerada no comportamento estrutural da laje a ocorrência de fissuração do concreto, o que torna o cálculo mais 
preciso. Com uma distribuição otimizada do reforço, pretende-se minimizar a quantidade de material, reduzindo-se os custos. Os resultados são comparados com as técnicas atualmente utilizadas no reforço de lajes de concreto armado com fibras de carbono.

\section{Modelo numérico das lajes reforçadas}

O procedimento básico de reforço à flexão de lajes usando compostos poliméricos é o de colar faixas ou tecidos nas regiões tracionadas da laje. As faixas cobrem partes da superfície da laje, enquanto os tecidos podem cobrir até a superfície completa da peça. A proposta deste trabalho é que, a partir das regiões determinadas pela otimização topológica, as faixas de fibras de carbono sejam aplicadas segundo uma ou duas direções, ou mesmo inclinadas, de forma similar à armação convencional com barras de aço.

Foram utilizados três tipos de materiais na confecção dos modelos numéricos:

(a) concreto (laje maciça);

(b) fibra de carbono (reforço); e

(c) aço (armaduras).

O reforço utilizado é um polímero reforçado por fibras de carbono (PRFC), da marca SikaCarbodur ${ }^{\odot}$, que tem as seguintes características: fibra de carbono em matriz epóxi com conteúdo de fibras em volume de $68 \%$; faixas com largura de $10 \mathrm{~cm}$ (adotado) e espessura de 1,2 $\mathrm{mm}$; módulo de elasticidade de $1,55 \times 10^{11} \mathrm{~N} / \mathrm{m}^{2}$; resistência à tração na ruptura de $3,1 \times 10^{9} \mathrm{~N} / \mathrm{m}^{2}$; e deformação máxima de 1,9\%; coeficiente de Poisson igual a 0,27 (adotado). O concreto utilizado tem as seguintes propriedades: resistência característica à compressão $f_{c k}=25 \mathrm{MPa}$; módulo de elasticidade secante $E_{c s}=2,38 \times 10^{10} \mathrm{~N} / \mathrm{m}^{2}$; e resistência à tração direta média $f_{c t, m}=2,56 \times 10^{6}$ $\mathrm{N} / \mathrm{m}^{2}$.
No que se refere à modelagem por elementos finitos, foram utilizados os seguintes elementos (ANSYS, 2010): fibra de carbono (reforço): SHELL63 - elemento de casca; fibra de carbono (reforço): SHELL93 - elemento de casca, específico para a otimização topológica; concreto (laje maciça): SOLID65 - elemento sólido; e aço (armaduras): BEAM3 - elemento de viga. A Figura 1 apresenta o esquema de modelagem por elementos finitos da laje reforçada.

Um aspecto importante no comportamento estrutural da laje e na determinação da região otimizada do reforço é a consideração da fissuração do concreto. O desenvolvimento das fissuras depende basicamente da geometria da laje (comprimento, largura e espessura), das condições de apoio, do posicionamento das armaduras e do carregamento aplicado. No programa de elementos finitos utilizado (ANSYS, 2010) a fissuração pode ocorrer em três direções ortogonais em cada ponto de integração do elemento, associadas às tensões principais $\sigma_{1}, \sigma_{2}$ e $\sigma_{3}$. A presença de uma fissura é representada pela modificação das relações tensãodeformação, introduzindo-se um plano de falha na direção normal à face da fissura. Quando a tensão principal no concreto excede a resistência à tração, a rigidez do elemento é reduzida para zero na direção principal, perpendicular ao plano fissurado. Para o caso de ocorrência apenas de tração em um estado multiaxial de tensões, tem-se $\sigma_{1} \geq \sigma_{2} \geq \sigma_{3} \geq 0$. O critério de falha do concreto, ou seja, de fissuração, é dado por $\sigma_{i}-f_{c t} \geq 0(i=1,2,3)$. Se o critério é satisfeito nas direções 1,2 e 3, a fissuração ocorre nos planos perpendiculares às tensões principais $\sigma_{1}, \sigma_{2}$ e $\sigma_{3}$. Se o critério é satisfeito nas direções 1 e 2 , a fissuração ocorre nos planos perpendiculares às tensões principais $\sigma_{l}$ e $\sigma_{2}$. Finalmente, se o critério é satisfeito somente na direção 1, a fissuração ocorre no plano perpendicular à tensão principal $\sigma_{l}$.

Figura 1 - Esquema de modelagem MEF para o procedimento de otimização topológica

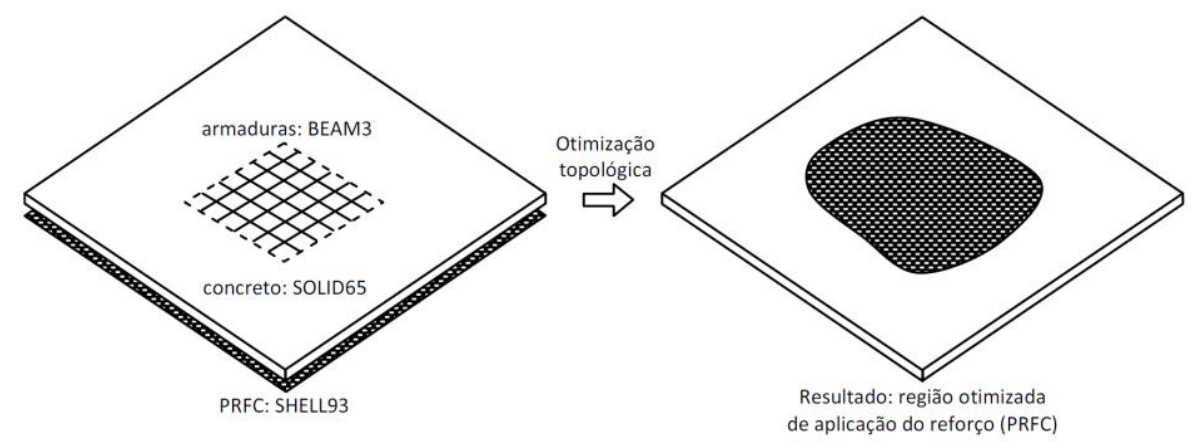


O coeficiente de transferência do cisalhamento $\beta_{t}$ representa a redução da resistência ao cisalhamento para as cargas subsequentes, que induzem deslizamento através da face da fissura. Se a fissura se fecha, as tensões de compressão normais ao plano da fissuração são transmitidas através da fissura, e apenas o coeficiente de transferência de cisalhamento $\beta_{c}$ para uma fissura fechada é introduzido. Os valores desses coeficientes de transferência de cisalhamento variam de 0 a 1 , onde 0 indica que não há transferência de cisalhamento e 1 indica que não há perda de transferência de cisalhamento. Foram feitas várias simulações em lajes para verificar a forma e o carregamento que dá início à formação de fissuras. Adotaram-se $\beta_{t}=0,3$ e $\beta_{c}=0,8$ (PADMARAJAIAH; RAMASWAMY, 2001).

A título ilustrativo, a Figura 2 mostra a distribuição de fissuras em uma laje engastada em dois bordos e apoiada em outros dois bordos. O carregamento utilizado na laje é aquele que vai gerar a formação significativa de fissuras. Esses carregamentos foram obtidos fazendo-se sucessivos acréscimos de carga no modelo de elementos finitos. Nota-se que as fissuras acompanham a distribuição esperada para os momentos fletores nas faces superior e inferior da laje.

A fim de validar quantitativamente o modelo de elementos finitos da laje, será avaliada a carga que provoca a fissuração do concreto. Para isso, deve- se utilizar combinação rara de ações. Nos casos em que não existem diversas ações variáveis de natureza diferente, o problema é simplificado. Em virtude das baixas tensões, admite-se que os módulos de elasticidade do concreto $\left(E_{c s}\right)$ e do aço $\left(E_{s}\right)$ sejam constantes e que a relação entre eles seja igual a 10 (Equação 1):

$\alpha_{e}=\frac{E_{s}}{E_{c s}}=10$

Eq. 1

$\mathrm{O}$ valor do momento que causa a primeira fissura, admitindo tensões baixas e diagrama linear, é (Equação 2):

$$
M_{r}=\alpha \frac{I}{y_{t}} f_{c t, m}
$$

Sendo:

$\alpha$ : coeficiente que depende da forma da seção transversal. Em lajes, a seção é retangular, $\operatorname{com} \alpha$ $=1,5$;

I: momento de inércia da seção bruta de concreto (inclusive armadura);

$y_{t}$ : distância da linha neutra à borda mais tracionada; e

$f_{c t, m}$ : resistência à tração do concreto.

A Figura 3 esquematiza a geometria da laje cuja carga de fissuração será calculada.

Figura 2 - Distribuição das fissuras em uma laje engastada/apoiada

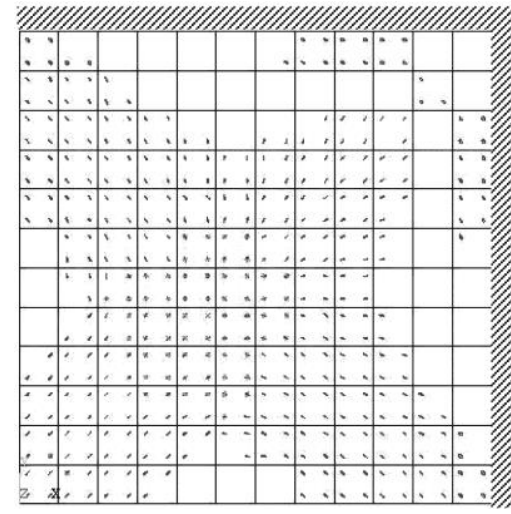

Face inferior da laje

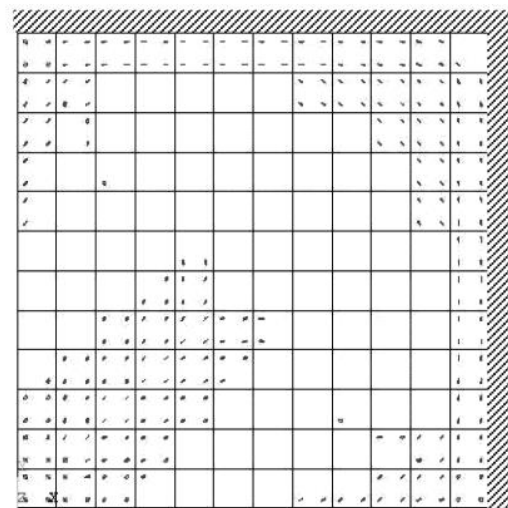

Face superior da laje

Figura 3 - Parâmetros de cálculo da carga de fissuração

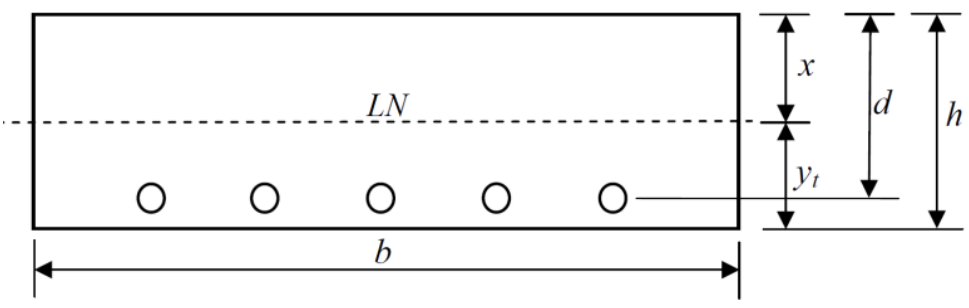

156 Cunha, J.; Chaves, L. P.; Santos, L. M. S. dos; Lopes, Z. M. F. 
O momento estático $M_{l}$ e a área $A$ valem (Eq. 3 e 4):

$$
\begin{aligned}
& M_{1}=\frac{b h^{2}}{2}+\left(\alpha_{e}-1\right) A_{1} d \\
& A=b h+\left(\alpha_{e}-1\right) A_{1}
\end{aligned}
$$

A posição da linha neutra $x$ é (Eq. 5):

$$
x=\frac{M_{1}}{A}
$$

Das Equações 3, 4 e 5, tem-se (Eq. 6):

$$
x=\frac{\frac{b h^{2}}{2}+\left(\alpha_{e}-1\right) A_{s} d}{b h+\left(\alpha_{e}-1\right) A_{1}}
$$

A Equação 7 fornece a inércia da seção fissurada:

$$
I=\frac{b x^{3}}{3}+\frac{b(h-x)^{3}}{3}+\left(\alpha_{e}-1\right) A_{s}(d-x)^{2}
$$

O momento $M_{r}$ que levará ao cálculo da carga de fissuração vale (Equação 8):

$$
M_{r}=C_{x} p l_{x}^{2}
$$

Onde o valor de $C_{x}$ é obtido da Tabela da Teoria da Elasticidade, em função de $\lambda$, sendo $\lambda=l_{x} / l_{y}\left(l_{x} \mathrm{e}\right.$ $l_{y}$ são os vãos da laje).

Como exemplo de cálculo da carga de fissuração de uma laje, supõe-se uma laje apoiada nos quatro bordos, submetida a um carregamento uniformemente distribuído. Têm-se os seguintes dados:

$h=10 \mathrm{~cm}$;

$l_{x}=l_{y}=5 \mathrm{~m} ; \mathrm{e}$

$f_{c k}=25 \mathrm{MPa} ;$ e $\mathrm{b}=100 \mathrm{~cm}$.

Substituindo esses dados nas equações de (1) a (6), resulta $x=5,05 \mathrm{~cm} ; y_{t}=4,95 \mathrm{~cm}$; e $I=8.493 \mathrm{~cm}^{4}$.

O momento de fissuração assume o valor $M_{r}=$ $6.580 \mathrm{~N} \times \mathrm{m}$. De acordo com a Tabela da Teoria da Elasticidade, tem-se para $\lambda=1, C_{x}=0,0368$, de onde o valor da carga (Equação 9):

$M_{r}=C_{x} p l_{x}^{2} \rightarrow 6.580=0,0368 \times p \times 5^{2} \rightarrow p=$ $7.150 \mathrm{~N} / \mathrm{m}^{2} \quad$ Eq. 9

Este carregamento é o que dará início à fissuração no concreto.

No programa computacional ANSYS foram feitas várias simulações da laje, verificando-se o carregamento que dá início à formação de fissuras, conforme abordagem e dados fornecidos no início desta seção. Foi encontrado o valor $p=7.240$ $\mathrm{N} / \mathrm{m}^{2}$. O erro relativo é da ordem de $1 \%$ ao se comparar com o resultado analítico obtido ( $p=$ $7.150 \mathrm{~N} / \mathrm{m}^{2}$ ), o que mostra que o cálculo feito pelo Modelo de Elementos Finitos é satisfatório.

\section{Formulação da otimização topológica do reforço}

O objetivo da otimização estrutural é a obtenção da estrutura mais resistente e mais rígida possível, com um menor custo. Isso deve ser feito pela mudança de configuração da peça dentro de um domínio de projeto especificado, com determinadas condições de carregamento e de contorno, estando em acordo com as condições de segurança.

Conforme já discutido, a otimização topológica determina a distribuição ótima de material no domínio de projeto. O objetivo é retirar ou redistribuir o material de maneira iterativa e sistemática. A distribuição ótima está relacionada com um objetivo, que pode ser, por exemplo, a minimização do volume final da estrutura ou a maximização da rigidez, visando à economia de material. Ela permite encontrar o leiaute estrutural ótimo, isto é, o número, a posição e o tamanho dos membros, além de vazios. Furos podem ser criados no domínio durante o processo de otimização. Assim, pode-se obter uma nova configuração da estrutura partindo-se apenas de definições básicas: domínio do projeto, condições de contorno e de carregamento, função objetivo, e restrições de projeto impostas.

A otimização topológica de estruturas contínuas é um tema relativamente recente no campo da otimização estrutural. O trabalho de Bendsoe e Kikuchi (1988) é um dos mais importantes neste tipo de otimização. A primeira etapa da otimização topológica em estruturas contínuas consiste em definir o domínio de projeto, as condições de contorno e as cargas aplicadas. Em uma segunda etapa, o domínio é discretizado por elementos finitos. Em seguida, as informações são inseridas no algoritmo de otimização, que, num processo iterativo, distribui o material no domínio fixado, de maneira a minimizar ou a maximizar a função objetivo, que pode ser, por exemplo, a maximização da rigidez, o que é equivalente à minimização da flexibilidade ou à minimização da energia de deformação da estrutura. O resultado obtido é uma estrutura com topologia (geometria) ótima. Na implementação numérica da otimização topológica, o modelo de elementos finitos que discretiza o domínio de projeto não é alterado durante o processo iterativo de otimização. Apenas a distribuição do material nos elementos é alterada. 
Na etapa iterativa de distribuição do material é necessário criar um modelo de material. Um método de modelagem e implementação computacional relativamente simples para o modelo de material é o Método das Densidades, que utiliza somente uma variável de projeto, a densidade relativa do material em cada elemento do domínio de projeto.

No Método das Densidades o domínio de projeto é discretizado por elementos finitos, que são preenchidos homogeneamente pelo material. A ideia é rearranjar o material trocando a densidade do material $(\rho)$ em cada elemento. Assim, obtêmse ao final do processo de otimização os elementos sólidos (densidade 1), onde há presença de material, e os elementos vazios (densidade 0), onde não há material. Graficamente, os resultados do procedimento iterativo de otimização indicam que elemento de cor branca tem alta flexibilidade (vazio); o elemento de cor preta tem baixa flexibilidade (material sólido); e o elemento de cor cinza tem flexibilidade intermediária (estado intermediário do material). A presença de cores intermediárias (cinzas) entre o preto e o branco não tem sentido prático, devendo ser eliminada. As variáveis de projeto são, portanto, as densidades dos elementos. No estágio final, as regiões de material sólido deverão formar os caminhos das cargas, enquanto as demais regiões estarão ocupadas por vazios.

A equação matemática que relaciona o valor da densidade em cada elemento do domínio, em função da propriedade efetiva do material $C(x)$ usado no projeto, é dada pelo Equação 10 (BENDSOE; SIGMUND, 2003):

$$
C(x)=\rho(x) C_{O}
$$

Onde $\rho(x)$ é uma função de distribuição contínua das densidades (variáveis de projeto), variando de 0 a 1 . O tensor $C_{0}$ pode ser, por exemplo, representado pelo módulo de Young $\left(E_{0}\right)$ do material. Para evitar a ocorrência de excesso de densidades intermediárias, estas serão penalizadas de acordo com a Equação 11:

$$
C(x)=\rho(x)^{\beta} C_{0},
$$

Sendo $\beta \geq 1$ o fator de penalização, que permite reduzir as densidades intermediárias no resultado final. Deve-se ter o cuidado de não tomar valores muito elevados para $\beta$, pois à medida que se aumenta esse valor, aproxima-se o problema contínuo para um problema discreto. O procedimento de penalização elimina com mais eficiência os elementos com densidades intermediárias, forçando a convergência para estados sólidos ou vazios.
O domínio de projeto é discretizado por elementos finitos, de onde podem ser obtidas as conhecidas expressões de equilíbrio da estrutura (Equação 12):

$K U=F$

Eq. 12

Sendo:

$K$ a matriz de rigidez global;

$U$ o vetor dos deslocamentos; e

$F$ o vetor das forças aplicadas.

A matriz de rigidez global $K$ resulta da soma das matrizes de rigidez dos elementos $K^{e}: K=\sum_{e=1}^{N} k^{e}$.

Onde $N$ é o número de elementos da estrutura discretizada. A variável de projeto $x^{e}$ representa a densidade relativa do material no elemento $e$, sendo $\rho=x^{e} \rho_{0}$. A densidade $\rho_{0}$ é a densidade de um elemento simples sólido do domínio $\left(x^{e}=1\right)$.

Nessa formulação, a grandeza a ser minimizada é a flexibilidade média da estrutura, que é equivalente à energia de deformação. Isso corresponde também a maximizar a rigidez global da estrutura. A flexibilidade média pode ser definida pela Equação 13 (BENDSOE; SIGMUND, 2003):

$S=F^{T} U=U^{T} K U=\sum_{e=1}^{N}\left(u^{e}\right)^{T} k^{e} u^{e}$

Eq. 13

Reescrevendo a Equação 11, que penaliza as densidades intermediárias, tem-se para um elemento (Eq. 14):

$k^{e}=\left(x^{e}\right)^{\beta} k^{0}$

Eq. 14

Sendo $\beta=3$ um valor indicado pela prática.

Introduzindo (14) em (13), a flexibilidade vale (Equação 15):

$S=\sum_{e=1}^{N}\left(x^{e}\right)^{\beta}\left[\left(u^{e}\right)^{T} k^{0} u^{e}\right]$

No processo de otimização, o volume $V(X)$ do material é fixado, sendo dado pela Equação 16:

$$
V(X)=x^{1} v_{1}+x^{2} v_{2}+\ldots+x^{N} v_{N}=\sum_{e=1}^{N} x^{e} v_{e}=X^{T} v
$$

Sendo:

$X$ o vetor das variáveis de projeto; e $v$ o vetor de volumes dos elementos.

Assim, pode-se equacionar o problema de otimização topológica como (SIGMUND, 2001):

Minimize: 
$S(X)=U^{T} K U=\sum_{e=1}^{N}\left(x^{e}\right)^{\beta}\left[\left(u^{e}\right)^{T} k^{0} u^{e}\right]:$ minimização da flexibilidade média da estrutura

Sujeita a:

$\frac{V(X)}{V_{0}}=f$ : restrição do volume do material

$K U=F \Rightarrow K U=\sum_{e=1}^{N}\left(x^{e}\right)^{\beta}\left[k^{o} u^{e}\right]=F$ : restrição de comportamento (equilíbrio da estrutura) $x_{\text {min }}^{e} \leq x^{e} \leq x_{\text {max }}^{e}, e=1, \ldots, N$ : restrições laterais,

Sendo:

$X=\left[\begin{array}{lllll}x^{1} & x^{2} & x^{3} & \ldots & x^{N}\end{array}\right]$ : vetor das variáveis de projeto (densidades dos elementos);

$K$ : matriz de rigidez global;

$U$ : vetor dos deslocamentos global;

$F$ : vetor das forças global;

$K^{0}$ : matriz de rigidez do elemento;

$u^{e}:$ vetor dos deslocamentos do elemento;

$N$ : número de elementos da estrutura discretizada;

$V_{0}$ : volume do domínio de projeto (volume inicial);

$f$ : fração de volume (redução desejada do volume de material);

$\beta$ : fator de penalização; e

$x_{\text {min }}^{e} ; x_{\text {max }}^{e}:$ limites inferior e superior das

variáveis de projeto $\left(x_{\text {max }}^{e}=1 ; x_{\text {min }}^{e}=10^{-3}\right.$ : este

valor serve para evitar a ocorrência de matriz de rigidez singular).

Neste trabalho o critério ou a função objetivo utilizada na otimização topológica do reforço das lajes consiste na maximização da rigidez global da estrutura, o que é equivalente à minimização da flexibilidade média ou, ainda, à minimização da energia de deformação. A topologia obtida pela maximização da rigidez pode levar a regiões onde há concentração de tensões, gerando valores muito elevados, o que ultrapassa os limites de resistência. No entanto, este não será um problema para o reforço de lajes com fibras de carbono proposto neste trabalho, porque o objetivo da otimização topológica será apenas de indicar as regiões onde se deve posicionar o reforço. Portanto, mesmo que ocorram violações dos limites de resistência do material, isso poderá ser corrigido num segundo momento, quando o resultado teórico da distribuição otimizada do reforço será ajustado para o uso prático no dimensionamento com faixas de PRFC.
Por outro lado, no reforço de lajes com fibras de carbono é importante, em termos do cálculo estrutural, que se maximize a resistência da estrutura reforçada. O ganho em termos de rigidez, com a diminuição da flecha, é relativamente pequeno quando se usam fibras de carbono, que possuem pequena espessura. Em contraponto à maximização da rigidez, que é um indicador global de desempenho, a maximização da resistência é uma medida local do desempenho da estrutura. Esta característica confere ao critério de máxima rigidez maior robustez do que o critério de máxima resistência, pois este último pode ter sua topologia influenciada por regiões onde existam concentrações de tensões ou ainda singularidades. Nesses casos, a topologia resultante é dominada por picos locais, conduzindo habitualmente a geometrias formadas por elementos concentrados, do tipo treliças e pórticos (DUYSINX, 1996). Embora não exista uma correspondência direta e precisa entre os resultados da otimização topológica obtidos segundo os critérios de maximização da resistência e da rigidez, alguns estudos indicam que, para muitos casos, há uma proximidade da geometria obtida (BENDSOE; DIAZ; KIKUCHI, 1993; LI; STEVEN; XIE, 1999; LAM; MANICKARAJAH; BERTOLINI, 2000).

O critério da máxima rigidez foi escolhido neste trabalho devido a sua robustez. Mesmo nos casos em que exista diferença entre a topologia otimizada pelos critérios da máxima rigidez e da máxima resistência, esta, em geral, não é significativa a ponto de mudar substancialmente a geometria do reforço, não levando, assim, a uma interferência significativa no resultado final de projeto. Neste estudo, o objetivo é localizar as regiões na superfície da laje onde se deve posicionar o reforço. Não se procura um resultado na otimização topológica que seja preciso e definitivo, para uso direto em projeto e dimensionamento. Assim, a partir das regiões a serem reforçadas (definidas pela otimização topológica), o dimensionamento será feito na sequência, utilizando-se faixas ou tecidos de fibra de carbono. Nesta segunda etapa, devem também ser considerados aspectos práticos do dimensionamento, como a necessidade de se acrescentar o comprimento de ancoragem ao reforço.

\section{Resultados obtidos com a otimização topológica}

O procedimento computacional utilizado para realizar a otimização topológica de reforço é composto das seguintes etapas gerais (ANSYS, 2010): definir o modelo de elementos finitos 
(malha, propriedades elásticas e de resistência dos materiais, carregamento e condições de apoio, parâmetros da fissuração); especificar as regiões a serem otimizadas: apenas os elementos identificados como tipo SHELL93 sofrem otimização topológica, o que corresponde ao PRFC colocado sobre a parte inferior e/ou superior da laje; definir a quantidade de material a ser removida (percentual " $V$ " de redução do volume de PRFC): $40 \%$ a $80 \%$; especificar a precisão da solução (convergência): $1 \times 10^{-4}$ ou número de iterações: 30; e impor restrições laterais, o que não é necessário para este tipo de problema. A Figura 4 esquematiza a sequência do procedimento de otimização topológica executada.

São apresentados a seguir os resultados das simulações de otimização topológica do reforço de lajes. Para a maioria os casos, a distribuição do reforço foi obtida com uma retirada de $64 \%$ de material, considerado inicialmente distribuído em toda a superfície da laje. O carregamento é uniformemente distribuído, sendo determinado para cada caso o valor que representa o estado fissurado da laje, variando de 7.000 a $19.000 \mathrm{~N} / \mathrm{m}^{2}$. $\mathrm{O}$ comprimento e a largura da laje valem $5 \mathrm{~m}$, e a espessura é de $10 \mathrm{~cm}$. Entre vários aspectos que influenciam o resultado da otimização topológica, foi avaliada a influência da fissuração, das condições de apoio e de carregamento, da quantidade de material e de duas configurações geométricas mais complexas. Para todos os resultados apresentados, a cor clara indica a região ótima onde o reforço deverá ser posicionado. As condições de apoio das lajes são representadas por traços contínuos, que indicam borda apoiada, traços pontilhados, que indicam borda livre, e hachuras, que indicam borda engastada.

Figura 4 - Procedimento de otimização topológica

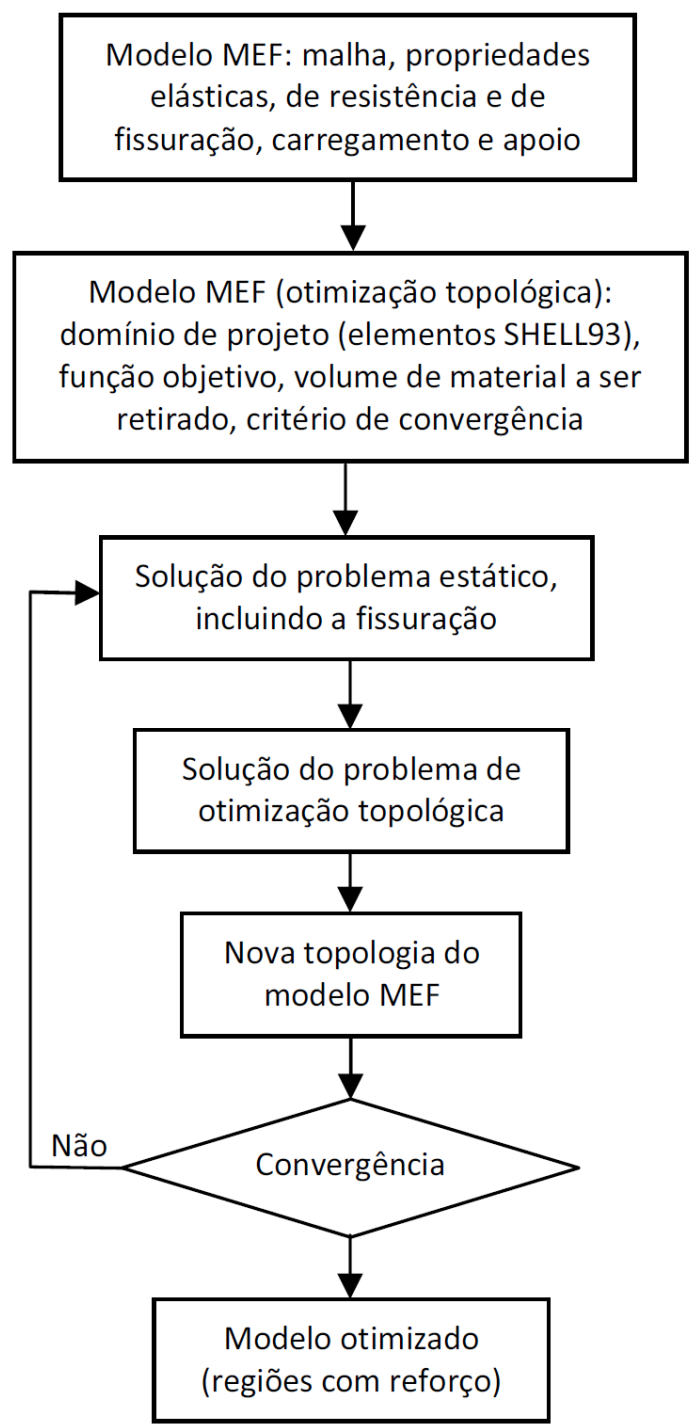

160 Cunha, J.; Chaves, L. P.; Santos, L. M. S. dos; Lopes, Z. M. F. 


\section{Influência da fissuração do concreto}

Conforme foi mencionado, a fissuração do concreto influencia o comportamento estrutural da laje, e por consequência, o resultado da otimização. A Figura 5 exemplifica a diferença da distribuição do reforço na face inferior da laje engastada em dois bordos e apoiada em outros dois, quando se considera a fissuração. Observa-se que há tendência de continuidade da distribuição otimizada do reforço na presença da fissuração.

\section{Influência das condições de apoio}

As condições de apoio têm influência significativa no comportamento estrutural da laje e, por consequência, na distribuição otimizada do reforço. A Figura 6 mostra a distribuição de reforço na face inferior (à esquerda) e na face superior (à direita) para diferentes condições de apoio. Nota-se que a distribuição do reforço nas faces acompanha a distribuição dos momentos fletores positivos e negativos. Nesse tipo de problema a otimização topológica não distingue as regiões tracionadas das comprimidas. Uma análise de tensões geradas permite essa verificação, visto que o reforço será executado apenas nas regiões tracionadas.

\section{Influência da quantidade de material de reforço}

Em função da necessidade do cálculo estrutural (dimensionamento), pode-se escolher a quantidade de reforço que será utilizada no procedimento de otimização topológica através do controle da quantidade de material a ser retirado. Foram consideradas nas simulações as retiradas de $40 \%$, $64 \%$ e $80 \%$ de material de reforço. Em função da quantidade de material, a geometria da distribuição do reforço será fortemente alterada, conforme mostra a Figura 7, para uma laje engastada nos quatro bordos.

\section{Figura 5 - Distribuição do reforço otimizado na presença de fissuração}

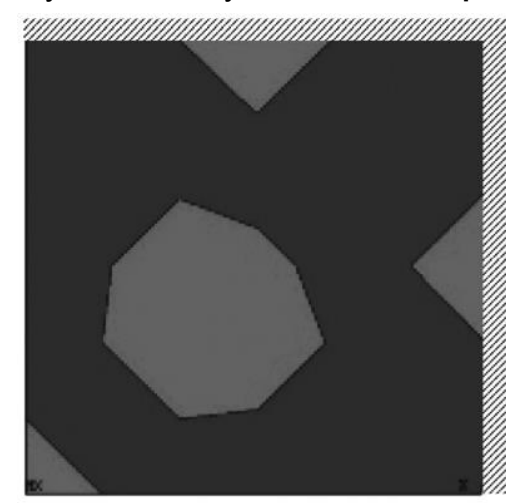

- Sem fissuração -

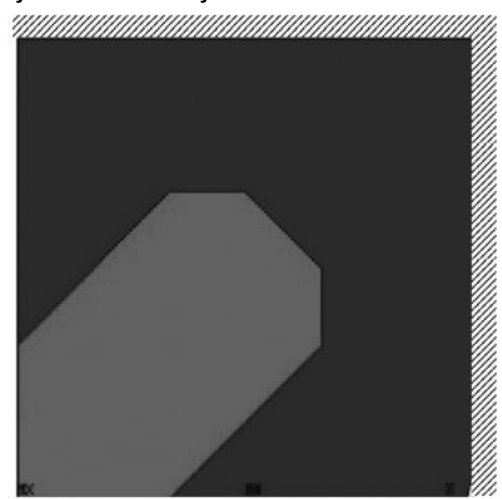

- Com fissuração - 
Figura 6 - Distribuição otimizada do reforço em função das condições de apoio
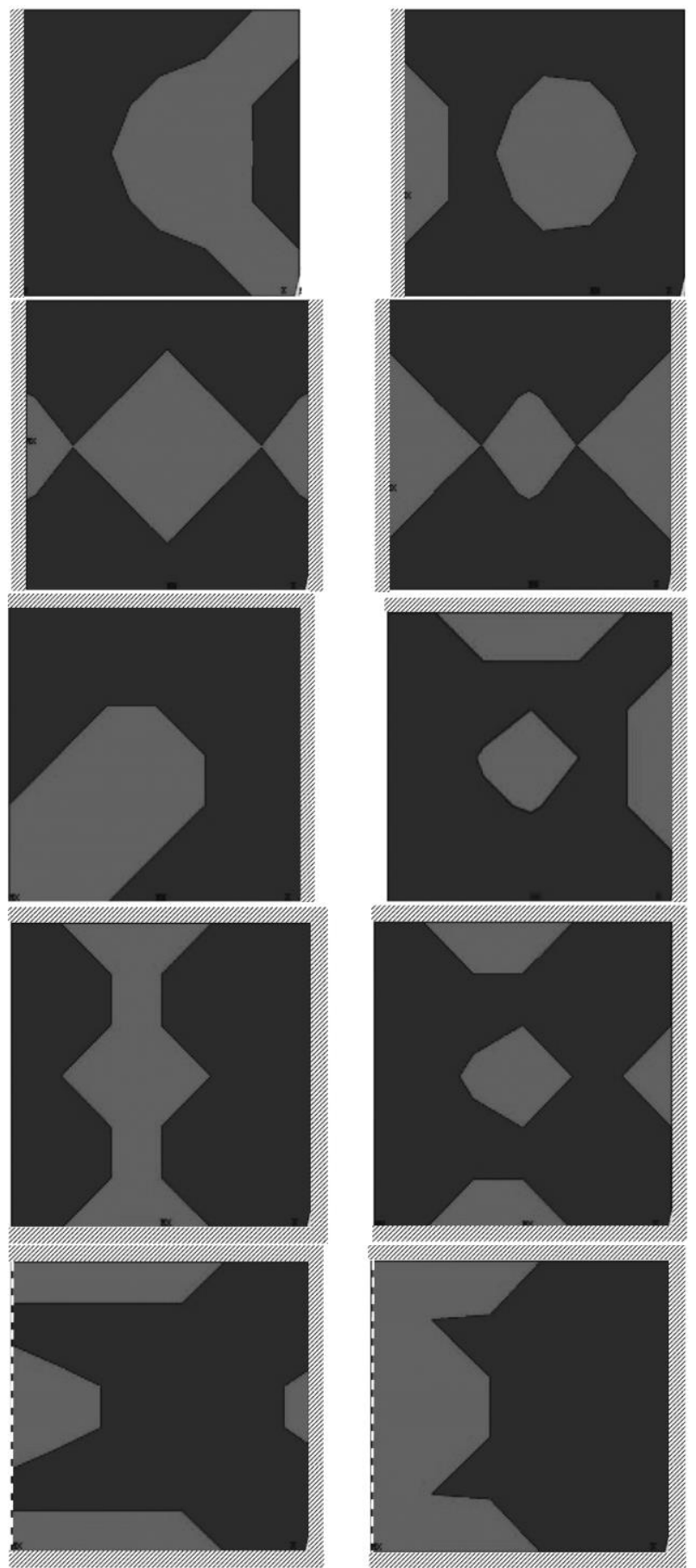

Figura 7 - Influência da quantidade de material retirado na distribuição otimizada do reforço
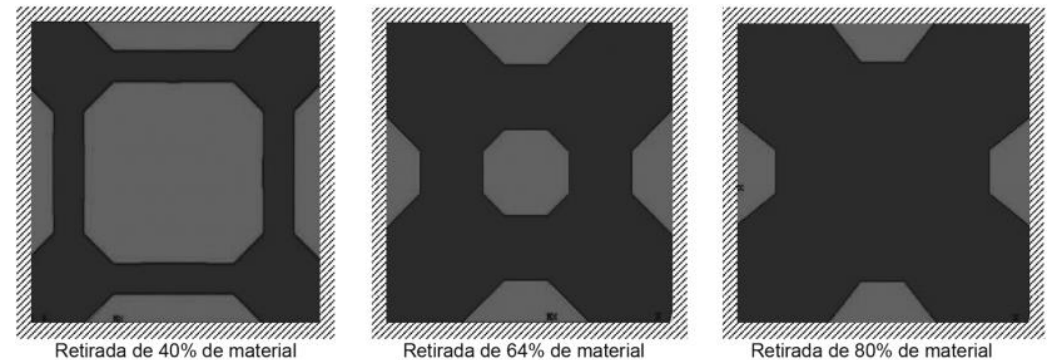

162 Cunha, J.; Chaves, L. P.; Santos, L. M. S. dos; Lopes, Z. M. F. 


\section{Influência do carregamento}

Para verificar a influência da forma de distribuição do carregamento no resultado otimizado do reforço, foram simuladas três situações de carregamento $\left(7.500 \mathrm{~N} / \mathrm{m}^{2}\right)$ para uma laje apoiada nos quatro bordos, conforme a Figura 8.

Percebe-se na Figura 9 a grande influência da forma de distribuição do carregamento no resultado otimizado do reforço.

\section{Reforço de lajes com configurações complexas}

Para avaliar o potencial dos resultados fornecidos pela otimização topológica, foi analisada a distribuição do reforço na face inferior de uma laje cogumelo e de uma laje triangular, que possuem uma situação estrutural mais complexa do que as lajes tradicionalmente projetadas. Estas lajes têm dimensões idênticas às anteriores. A laje cogumelo está apoiada em quatro pilares, distantes $90 \mathrm{~cm}$ das bordas.

A Figura 10 mostra que a distribuição do reforço na laje cogumelo pode mudar significativamente em função das dimensões dos pilares $(20 \mathrm{~cm}$ x 20 $\mathrm{cm}$ no caso a, e $20 \mathrm{~cm}$ x $80 \mathrm{~cm}$ no caso b). Além das regiões tradicionais de aplicação do reforço, onde ocorre o efeito de punção, foram indicadas também regiões entre os pilares.

Figura 8 - Formas de distribuição do carregamento para uma laje apoiada

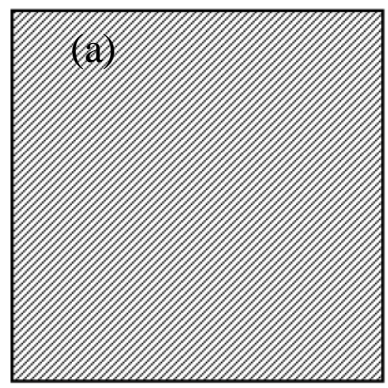

- Em toda a laje -

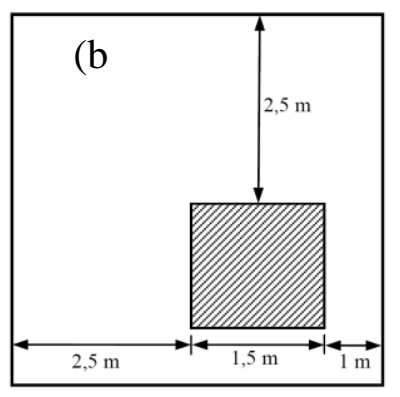

- Distribuído parcialmente -

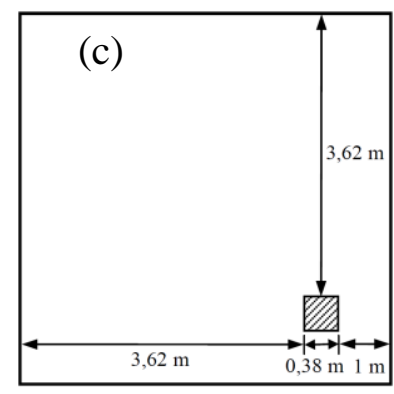

- Concentrado numa região -

Figura 9 - Regiões do reforço otimizado sob diferentes carregamentos

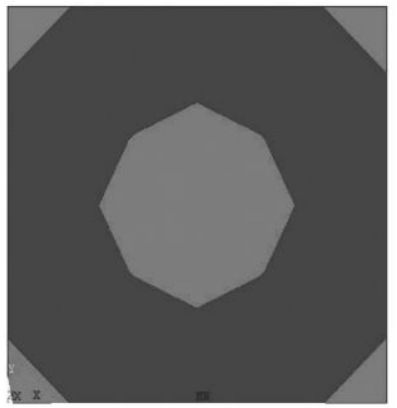

(a)

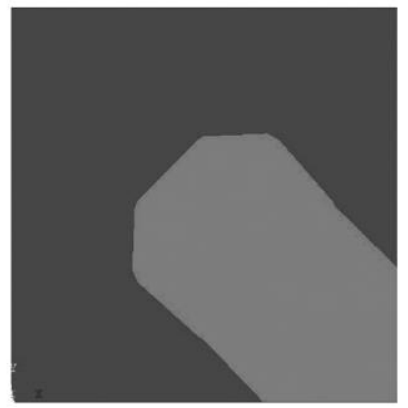

(b)

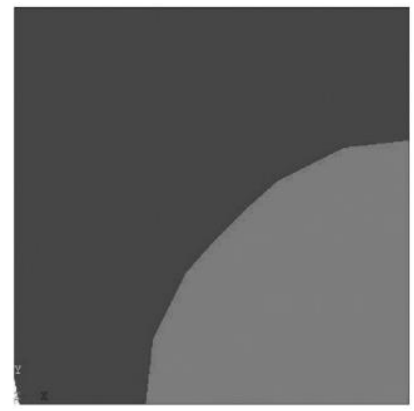

(c)

Figura 10 - Distribuição otimizada do reforço para uma laje cogumelo, considerando diferentes dimensões dos pilares

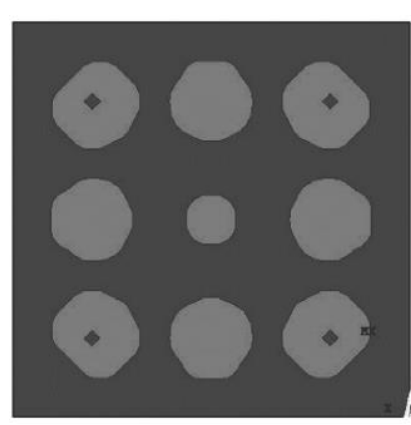

(a)

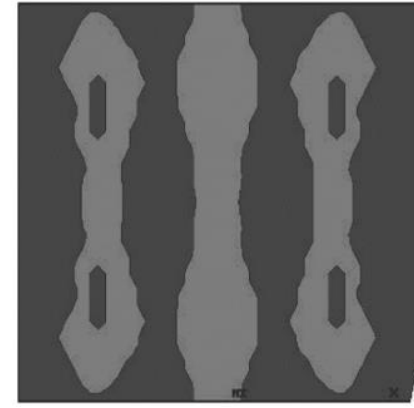

(b) 
A Figura 11 explicita a influência das condições de apoio na distribuição do reforço para a laje triangular. Assim como nos casos anteriores, as regiões indicadas de reforço acompanham a distribuição dos momentos fletores positivos e negativos.

Considerando o resultado obtido para a localização e a forma das regiões de reforço desses dois casos, pode-se supor que haveria dificuldade em encontrar de maneira eficiente os mesmos resultados com as técnicas tradicionais de dimensionamento.

\section{Comparação do reforço otimizado com o reforço convencional}

A fim de verificar o desempenho da distribuição otimizada do reforço, são apresentados resultados do comportamento estrutural (resistência e rigidez) para a laje sem reforço, com reforço no modo convencional (como é feito na prática) e com reforço distribuído segundo os resultados fornecidos pela otimização topológica. Para isso, o dimensionamento do reforço é feito no estado limite último. Todos os carregamentos foram considerados como uniformemente distribuídos sobre as lajes. Para o concreto foi empregado o diagrama tensão-deformação idealizado, em que, para tensões de compressão menores que metade da resistência à compressão, pode-se admitir uma relação linear entre tensões e deformações. Para o cálculo nos estados limites de serviço e último do aço utilizou-se também o diagrama tensãodeformação específica simplificado, em que se prevê relação linear entre tensão e deformação específica.

Uma vez dimensionado o reforço de PRFC, ele é distribuído na face da laje segundo o modo convencional, que é aquele que, pelo menos por hipótese, é feito na prática. A seguir, calcula-se a área percentual desse reforço em relação à área total da laje. Esse valor será em seguida utilizado no procedimento de otimização topológica para indicar a quantidade de material a ser retirado. Como resultado, a otimização topológica mostrará a região onde o reforço deverá ser colocado. Nesta região a geometria de distribuição do reforço poderá ser similar à do modo convencional. Isso quer dizer que a otimização topológica será utilizada principalmente como uma ferramenta de localização da região ótima para posicionamento do reforço, visando à economia de material.

Figura 11 - Distribuição otimizada do reforço para uma laje triangular em diferentes condições de apoio
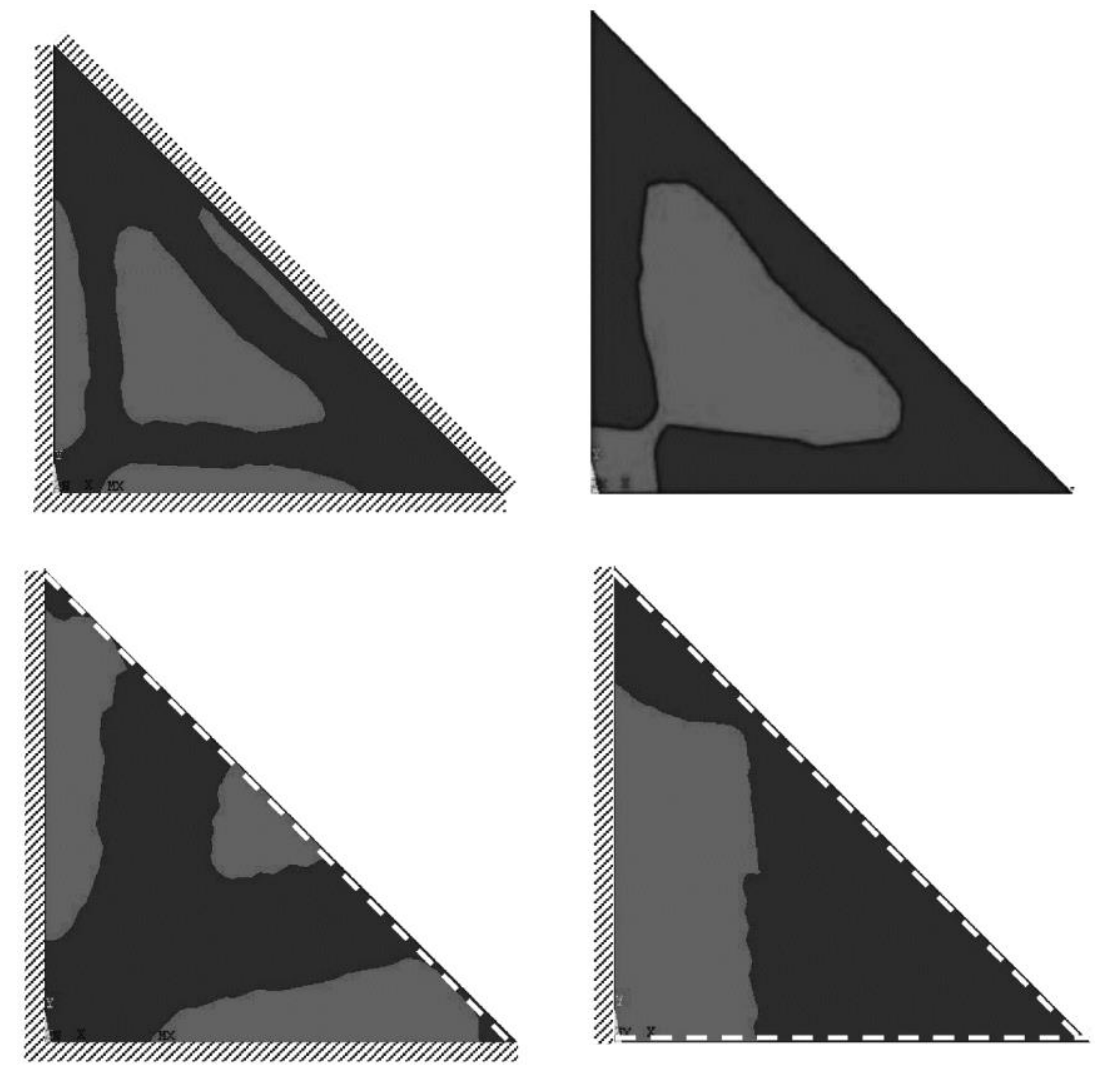

164 Cunha, J.; Chaves, L. P.; Santos, L. M. S. dos; Lopes, Z. M. F. 
No projeto de reforço à flexão se consideram, fundamentalmente, os mesmos princípios que se empregam em reforços mediante chapas de aço equilíbrio da seção, compatibilidade de deformações específicas e hipóteses NavierBernoulli -, onde as seções planas permanecem planas após deformação. Com o objetivo de se obter uma resposta eficiente, deve-se projetar o reforço de modo que a ruptura do elemento em PRFC ocorra durante a plastificação do aço e antes que o concreto alcance a ruptura por compressão. Para o PRFC utilizado neste trabalho, deve-se prever no dimensionamento uma deformação específica limite do reforço de 7,6\%. Além disso, a tensão limite de tração no reforço não deve ultrapassar $2,28 \times 10^{9} \mathrm{~N} / \mathrm{m}^{2}$. O dimensionamento do reforço no estado limite último foi feito segundo o ACI 440.2R (AMERICAN..., 2003). Os detalhes podem ser encontrados em Chaves (2010). O resultado do cálculo da área de reforço é dividido pela espessura, o que fornece a largura necessária por metro de laje. Em seguida, acrescenta-se o comprimento de ancoragem às faixas de reforço.

A laje simulada está apoiada em dois bordos e engastada nos outros dois bordos, com comprimento e largura de $5 \mathrm{~m}$, espessura de 10 $\mathrm{cm}$, e altura útil de $8 \mathrm{~cm}$. O carregamento inicial é uniformemente distribuído e vale $7.000 \mathrm{~N} / \mathrm{m}^{2}$. Para promover o aumento da capacidade de carga, o reforço será dimensionado para que a laje suporte um carregamento final de $19.000 \mathrm{~N} / \mathrm{m}^{2}$. Por questões práticas, considerou-se que o reforço será colocado apenas na face inferior das lajes, nas regiões tracionadas. A justificativa é a de que, em função do tipo de utilização da laje, a face superior pode ser de difícil acesso e execução do reforço.

$\mathrm{O}$ resultado obtido para a distribuição convencional do reforço é apresentado na Figura 12. Nesta distribuição há quatro faixas horizontais e quatro verticais, com comprimento de $390 \mathrm{~cm} \mathrm{e}$ largura de $10 \mathrm{~cm}$, espaçadas de $90 \mathrm{~cm}$, resultando numa área de reforço de $3,12 \mathrm{~m}^{2}$.

A Figura 13 destaca a região tracionada na face inferior da laje encontrada pela otimização e, em seguida, a distribuição do reforço em faixas nesta região através de uma seção quadrada de lado igual a $170 \sqrt{2} \mathrm{~cm}$. A opção de adotar faixas inclinadas a $45^{\circ}$ veio da observação da geometria da área otimizada e da análise do comportamento estrutural da laje. A área de reforço neste caso é de $1,70 \mathrm{~m}^{2}$, e a largura de cada faixa é de $10 \sqrt{2} \mathrm{~cm}$, valor não inteiro, próximo de $14 \mathrm{~cm}$. A justificativa de se adotar esse modelo ajustado é facilitar o encaixe do reforço na malha de elementos finitos. Percebe-se redução significativa de área de reforço otimizada quando comparada com a distribuição convencional.

Para comparar quantitativamente o desempenho da distribuição otimizada do reforço, foram calculadas tanto as tensões na laje reforçada, que indicam o comportamento em termos de resistência, quanto as flechas, um indicativo do comportamento em termos de rigidez. Os resultados são apresentados na Tabela 1 .

Ao se compararem as tensões normais $\sigma_{x}$ e $\sigma_{y}$ e as tensões de Von Mises $\sigma_{V M}$ nas três situações, verifica-se redução considerável na presença de reforço. Os resultados obtidos para o reforço otimizado são próximos do convencional, mas com uma área de reforço bem menor, cerca de $45 \%$ inferior. A flecha teve pequena redução, o que já era esperado, pois o reforço com fibras de carbono, que possuem pequena espessura, não tem influência significativa na diminuição desse efeito.

Essa simulação mostra que é possível reforçar a laje com uma área de reforço bem menor do que aquela que, em princípio, seria feita tradicionalmente. Como consequência, há economia de material.

Figura 12 - Distribuição das faixas de reforço no modo convencional

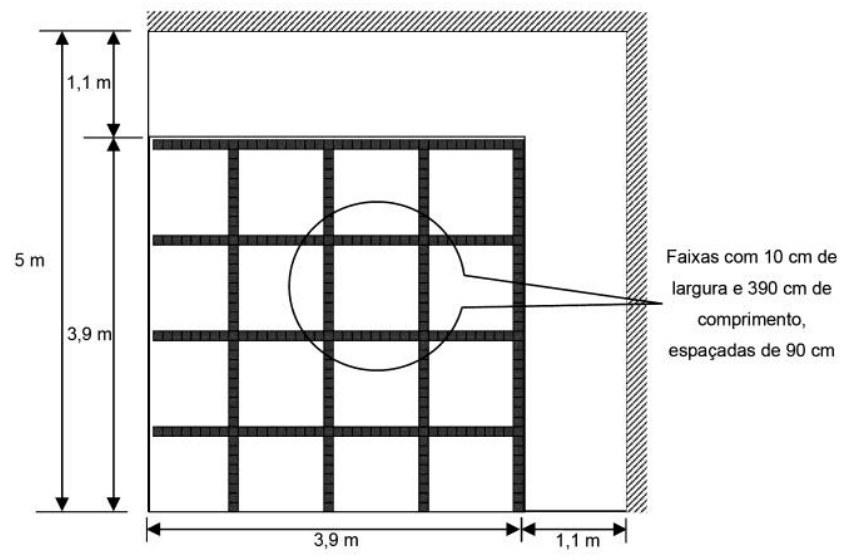


Figura 13 - Região de reforço obtida pela otimização topológica e distribuição otimizada do reforço

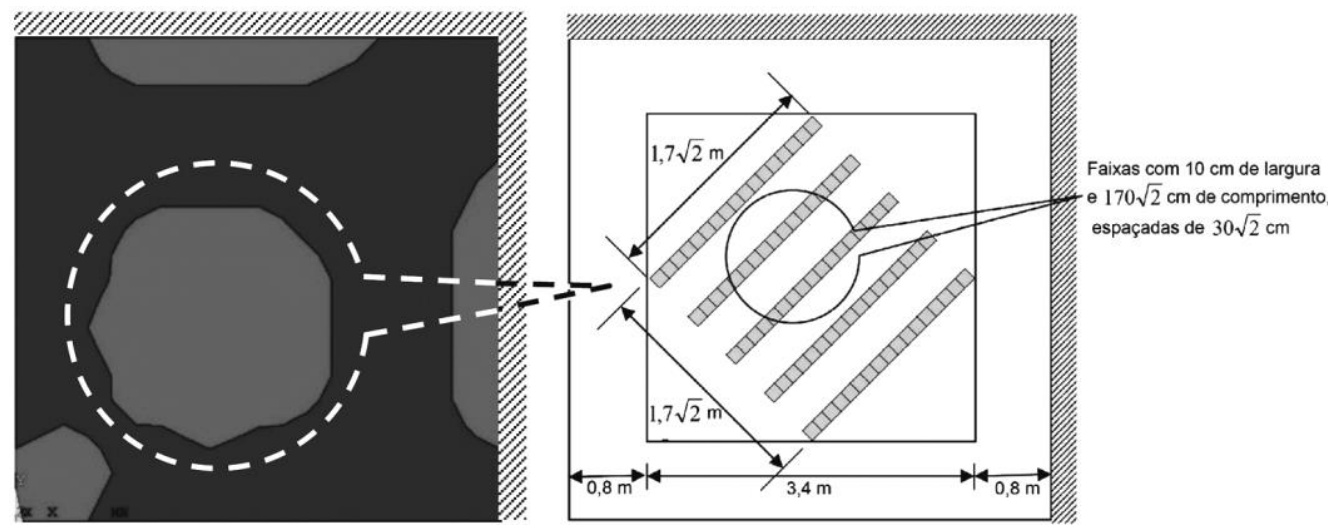

Tabela 1 - Tensões e flechas da laje reforçada de forma convencional e otimizada

\begin{tabular}{|c|c|c|c|c|c|c|}
\hline & \multicolumn{2}{|c|}{$\sigma_{x}=\sigma_{y}\left(x 10^{6} \mathrm{~N} / \mathrm{m}^{2}\right)$} & \multicolumn{2}{|c|}{$\sigma_{V M}\left(x 10^{6} \mathrm{~N} / \mathrm{m}^{2}\right)$} & \multirow[b]{2}{*}{$\begin{array}{l}\text { Flecha } \\
\text { máxima } \\
(\mathbf{m m})\end{array}$} & \multirow{2}{*}{$\begin{array}{c}\text { Área de } \\
\text { reforço } \\
\left(\mathbf{m}^{2}\right)\end{array}$} \\
\hline & $\begin{array}{c}\text { Ponto } \\
(1,9 ; 1,9 ; 0) \\
\text { face inferior }\end{array}$ & $\begin{array}{c}\text { Ponto } \\
(1,9 ; 1,9 ; 0,10) \\
\text { face superior }\end{array}$ & $\begin{array}{c}\text { Ponto } \\
(1,9 ; 1,9 ; 0) \\
\text { face inferior }\end{array}$ & $\begin{array}{c}\text { Ponto } \\
(1,9 ; 1,9 ; 0,10) \\
\text { face superior }\end{array}$ & & \\
\hline $\begin{array}{l}\text { Sem reforço } \\
\text { Convencional } \\
\text { Otimizado }\end{array}$ & $\begin{array}{l}8,45 \\
7,14 \\
6,49\end{array}$ & $\begin{array}{l}-8,53 \\
-8,01 \\
-7,90\end{array}$ & $\begin{array}{l}8,46 \\
7,15 \\
6,71\end{array}$ & $\begin{array}{l}8,53 \\
7,90 \\
7,72\end{array}$ & $\begin{array}{c}12,58 \\
7,90 \\
7,72\end{array}$ & $\begin{array}{c}\cdots \\
3,12 \\
1,70\end{array}$ \\
\hline
\end{tabular}

\section{Conclusões}

A partir das simulações numéricas realizadas, pode-se concluir que a otimização topológica mostrou-se uma ferramenta muito interessante para a determinação da distribuição do reforço, especialmente porque possibilita economia de material. Embora neste trabalho a técnica tenha sido aplicada em lajes, o procedimento pode ser usado em qualquer estrutura de concreto armado a ser reforçada.

Em alguns casos, a solução otimizada pode ser inabitual em relação à forma convencional de reforço, o que indica a otimização como um recurso bastante útil. De fato, para casos que apresentem geometrias diferenciadas (curvas, aberturas, estruturas tridimensionais, etc.) e condições de apoio e de carregamento particulares, a determinação da distribuição do reforço na forma convencional pode não ser evidente.

As simulações mostraram que diversos fatores podem afetar o resultado da otimização, como a fissuração do concreto, a taxa de retirada de material e as condições de apoio e de carregamento. Assim, para cada caso deve ser determinada a região otimizada, aplicando-se nela o reforço calculado no estado limite último.

\section{Referências}

AGBOSSOU, A. et al. Strengthening Slabs Using Externally-Bonded Strip Composites: analysis of concrete covers on the strengthening. Composites: Part B, v. 39, p. 1125-1135, 2008.

\section{AMERICAN CONCRETE INSTITUTE. ACI}

440.2R: guide for the design and construction of externally bonded FRP systems for strengthening concrete structures. Detroit, MI: ACI, 2003.

ANSYS 12.0 Notice. Computational Applications and System Integration Inc., 2010.

BENDSOE, M. P.; DIAZ, A.; KIKUCHI, N. Topology and Generalized Layout Optimization of Elastic Structures. In: BENDSOE, M. P.; MOTA SOARES, C. A. (Eds.). Topology Design of Structures. Kluwer Dordrecht, 1993. (Proc NATO ARW, Sesimbra 1992).

BENDSOE, M. P.; KIKUCHI, N. Generating Optimal Topologies in Structural Design Using a Homogenization Method. Computer Methods in Applied Mechanics and Engineering, v. 71, n. 2, p. 197-224, 1988.

BENDSOE, M. P.; SIGMUND, O. Topology

Optimization: theory, methods and applications. Berlin: Springer-Verlag; 2003.

BRUGGI, M. Generating Strut-and-Tie Patterns For Reinforced Concrete Structures Using Topology Optimization. Computers and Structures, v. 87, n. 23/24, p. 1483-1495, 2009. 
CHAVES, L. P. Estudo da Distribuição do Reforço de Lajes de Concreto Armado Com Fibras de Carbono, Usando Otimização Topológica. 122 f. Uberlândia, 2010. Dissertação (Mestrado em Engenharia Civil) - Faculdade de Engenharia Civil, Universidade Federal de Uberlândia, Uberlândia, 2010.

DUYSINX, P. Optimisation Topologique: du milieu continu à la structure élastique. 1996. Thèse (Doctorat) - Université de Liège, 1996.

EBEAD, U.; MARZOUK, H.; LYE, L. M. Strengthening of Two-Way Slabs Using FRP Materials: a simplified analysis based on response surface methodology. In: WORLD

ENGINEERING CONGRESS, 2., Sarawak, Malaysia, 2002. Proceedings... Sarawak, Malaysia, 2002.

GUAN, H. et al. Bridge Topology Optimization With Stress, Displacement and Frequency Constraints. Computers and Structures, v. 81, n. 3, p. 131-145, 2003.

HOLLAWAY, L. C.; HEAD, P. R. Advanced Polymer Composites and Polymers in the Civil Infrastructure. Amsterdam \& London: Elsevier Science; 2001.
LAM, Y. C.; MANICKARAJAH, D.; BERTOLINI, A. Performance Characteristics of Resizing Algorithms For Thickness Optimization of Plate Structures. Finite Elements in Analysis and Design, v. 34, n. 2, p. 159-174, 2000.

LI, Q.; STEVEN, G. P.; XIE, Y. M. On Equivalence Between Stress Criterion and Stiffness Criterion in Evolutionary Structural Optimization. Structural Optimization, v. 18, n. 1, p. 67-73, 1999.

MICHEL, L. et al. Flexural Stiffness Modelling of RC Slab Strengthened By Externally Bonded FRP. Composites: Part B, v. 40, n. 8, p. 758-765, 2009.

MOSALLAM, A. S.; MOSALAM, K. M. Strengthening of Two-Way Concrete Slabs With FRP Composite Laminates. Construction and Building Materials, v. 17, p. 43-54, 2003.

PADMARAJAIAH, S. K.; RAMASWAMY, A. A Finite Element Assessment of Flexural Strength of Prestressed Concrete Beams With Fiber Reinforcement. Civil Engineering Department, Indian Institute of Science, India, 2001.

SIGMUND, O. A 99 Line Topology Optimization Code Written in Matlab. Structural and Multidisciplinary Optimization, v. 21, n. 2, p. 120-127, 2001

\footnotetext{
Jesiel Cunha

Faculdade de Engenharia Civil | Universidade Federal de Uberlândia | Av. João Naves de Ávila, 2121, Campus Santa Mônica, Santa Mônica | Uberlândia - MG - Brasil | CEP 38400-902 | Tel.: (34) 3239-4446 | E-mail: jecunha@ufu.br

Luciano Pereira Chaves

Faculdade de Engenharia Civil | Universidade Federal de Uberlândia | E-mail: lucianopereirach@yahoo.com.br

Leila Maria Soares dos Santos

Departamento de Engenharia e Ciências do Mar | Universidade de Cabo Verde | Praça António Lereno - Praia | Santiago - Cabo Verde | CP 379C | Tel.: + (238) 334-0200 | E-mail: lessantos88@hotmail.com

Zigue Marley Furtado Lopes

Departamento de Engenharia e Ciências do Mar | Universidade de Cabo Verde | E-mail: ziggks@hotmail.com
}

Revista Ambiente Construído

Associação Nacional de Tecnologia do Ambiente Construído

Av. Osvaldo Aranha, 99 - $3^{\circ}$ andar, Centro

Porto Alegre - RS - Brasil

CEP $90035-190$

Telefone: +55 (51) 3308-4084

Fax: +55 (51) 3308-4054

www.seer.ufrgs.br/ambienteconstruido

E-mail: ambienteconstruido@ufrgs.br 